\title{
Variation of Left Atrial Function in Different Stages of Mitral Regurgitation and Its Association With Guidelines-Based Surgical Indication
}

\author{
Fengming $\mathrm{Bai}^{*}{ }^{1}$ Lingfei Cui ${ }^{*}{ }^{2} \mathrm{Bo} \mathrm{Li}^{2}$ \\ ${ }^{1}$ Nanxishan Hospital of Guangxi Zhuang Autonomous Region, Guangxi Zhuang Autonomous Region Second People's Hospital, \\ Guilin, Guangxi, China; ${ }^{2}$ Seventh Affiliated Hospital of Sun Yat-Sen University, Shenzhen, China
}

\section{ABSTRACT}

Purpose: The current guidelines associate indications for surgery in mitral regurgitation (MR) with left ventricle size and function. However, there is not enough emphasis in current guidelines on left atrial function, which is thought to be an important factor predicting adverse outcomes in MR. The aim of this study was to investigate the left atrial function at different stages of mitral regurgitation and its value in predicting the indications of mitral valve surgery.

Methods: This was a retrospective study with 163 consecutive chronic primary MR patients who underwent color doppler echocardiography at the Guangxi Zhuang Autonomous Region Second People's Hospital between January 2016 and June 2018. All patients were in sinus rhythm, classified into three groups, according the degree of mitral regurgitation. Comparison was made with 30 control patients. Using Simpson's methods, we recorded maximal left atrial volume, left atrial volume before active contraction and minimal left atrial volume, from which left atrial expansion index, left atrial passive emptying fraction, left atrial active emptying fraction, and the total left atrial emptying fraction were derived.

Results: Left atrial volume was expanded and left atrial emptying fraction was reduced in the mitral regurgitation group. By multivariate analysis, left atrial passive emptying fraction and left atrial active emptying fraction were independent predictors of mitral regurgitation requiring surgery. Using receiver-operating characteristic analysis, left atrial passive emptying fraction $<97.4 \%$ demonstrated $98 \%$ sensitivity and $67 \%$ specificity for predicting the presence of surgical indication (area under the curve: $0.91 ; P<.001$ ).

Conclusion: During mitral regurgitation, left atrial volume increases and functions decrease. The left atrial passive emptying fraction can be used as an additional tool to predict the indications of mitral valve surgery.

Received Fuly 14, 2020; accepted August 26, 2020.

*Both authors contributed equally to this work

Correspondence: Bo Li; Seventh affiliated Hospital of Sun Yat-Sen University, No. 628, Zhenyuan Road, Xinhu Street, Guangming District, Shenzhen, China; +8619129539810 (e-mail: libo78@mail.sysu.edu.cn).

\section{INTRODUCTION}

Mitral regurgitation (MR) is the most common valvular disease and likely to increase over the next several decades given an aging population [Nkomo 2006; Nishimura 2017]. Without surgical intervention, continued disease progression and constrained prognosis will occur in patients with MR [Avierinos 2002; Enriquez-Sarano 2005]. The optimal timing of surgical intervention in patients with $\mathrm{MR}$ is of paramount importance, and it has been continuously revised, however, remains controversial [Kang 2014]. The current guidelines associate indications for surgery in mitral regurgitation (MR) with left ventricle $(\mathrm{LV})$ size and function. However, there is not enough emphasis in current guidelines on left atrial function, which is thought to be an important factor predicting adverse outcomes in MR [Le Tourneau 2010].

In primary $M R$, the LA and $L V$ are subject to increased preload. Despite this, LV ejection fraction (LVEF), a traditional predictor, can remain normal or enhanced for a long time [Delahaye 1991]. The LV can pump excess blood into the low-pressure LA and result in increased LA pressure and volume load. This makes the LA the most severely affected cardiac chamber, with functional impairment at the early stage of MR. Changes in left atrial volume (LAV) and function may be earlier and substantial than those in the $\mathrm{LV}[\mathrm{Li}$ 2018], so an in-depth study of LA function and size may provide deeper insight into the pathophysiology of $M R$ and a reference for disease stage.

According to the volume change of the LA, the LA shows three processes: passive expansion, passive contraction, and active contraction. The ability of atrial deformation in these three processes can reflect the left atrial function. The aim of this study was to investigate the left atrial volume and function at different stages during MR and its association with guidelines-based surgical indication.

\section{MATERIALS AND METHODS}

Patient population: We studied consecutive chronic primary MR patients who underwent color doppler echocardiography at the Guangxi Zhuang Autonomous Region Second People's Hospital between January 2016 and June 2018. Patients with the following conditions were excluded. These include atrial fibrillation, coexistent mitral stenosis or aortic valve disease, other organic heart disease, significant 
a

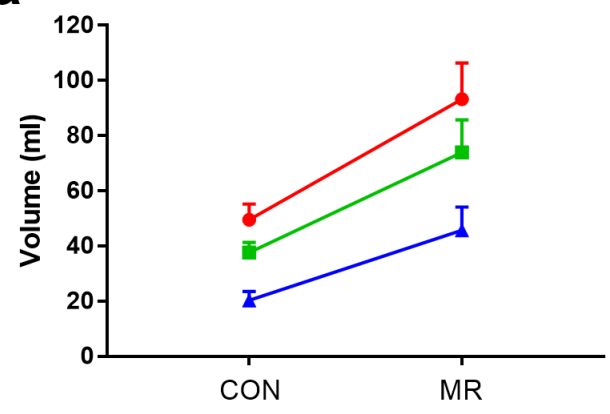

C

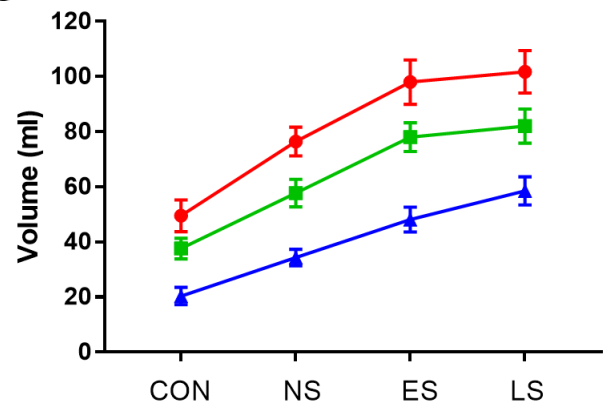

b
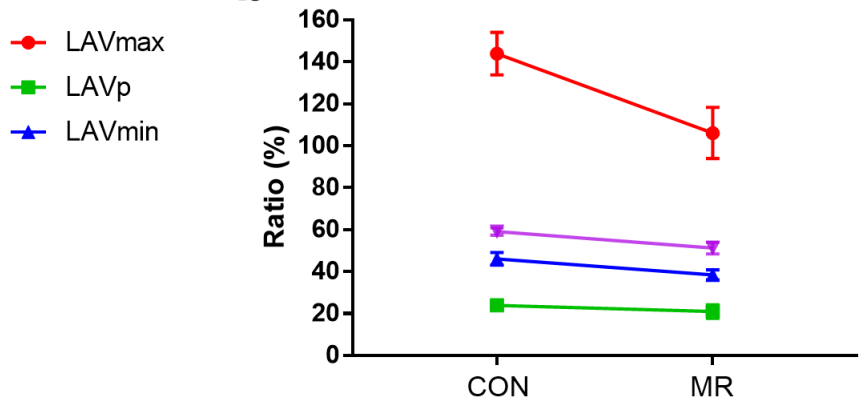

$\rightarrow$ LAEI

$\rightarrow$ LAPEF

$\rightarrow$ LAAEF

$\rightarrow$ TLAEF

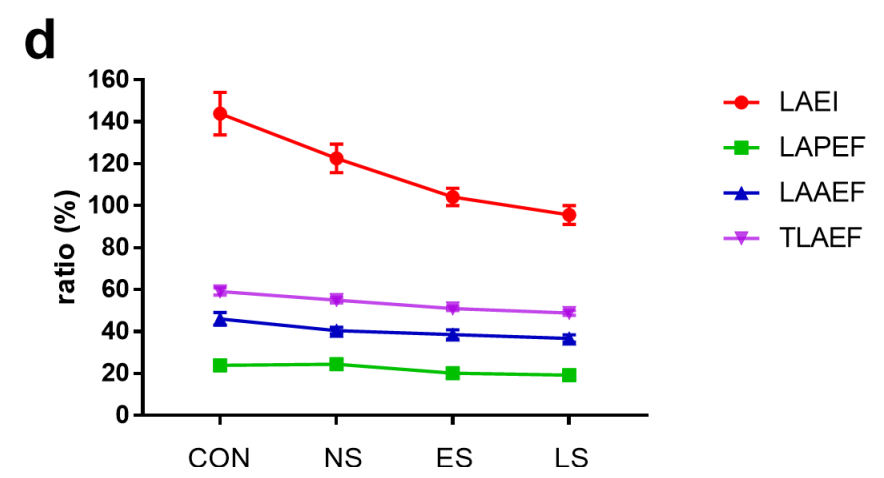

Figure 1. Comparison of left atrial volume (A) and function (B) between mitral regurgitation group and control group; comparison of left atrial volume (C) and function (D). CON: control group; NS: non-severe group; ES: early-severe group; LS: late-severe group

pulmonary disease, and poor acoustic windows. Patients were classified into: mild to moderate MR (non-severe group, $\mathrm{N}$ = 38); severe $M R$ without surgical indication (early-severe group, $\mathrm{N}=43$ ); and severe MR with at least one surgical indication (late-severe group, $\mathrm{N}=52$ ). Early-stage of severe MR was defined as the absence of established indications for surgical intervention, according to American guidelines, including rational symptoms, left ventricular end-systolic diameter (LVESV) $\geq 40 \mathrm{~mm}$, left ventricular ejection fraction (EF) $<60 \%$, or pulmonary artery pressure $>50 \mathrm{mmHg}$ at rest or $60 \mathrm{mmHg}$ with exercise, while late-stage of severe MR was defined as a combination of one or more items. Comparison was made with a group of 38 patients without cardiovascular disease, and the parameters of echocardiography were in the normal range. The patients in the study were informed of the purpose of the study, and informed consent was obtained. The study was approved by the ethics committee of the Nanxishan Hospital of Guangxi Zhuang Autonomous Region. The methods in the study were carried out in accordance with the relevant guidelines and regulations.

Echocardiography: Echocardiography was performed using an iE33 echocardiograph (Philips, Andover, Massachusetts). The recommendations of the American Society of Echocardiography were used to determine the severity of MR, volume of LA and LV. The ratio of the regurgitation area to left atrial area (RA/LAA) was used to evaluate MR: greater than $40 \%$ indicated severe regurgitation, less than $20 \%$ mild regurgitation, and between $20 \%$ and $40 \%$ moderate regurgitation. The LAVmax (defined as the volume immediately prior to mitral valve opening), the $\mathrm{LAVp}$ (defined as the volume at $\mathrm{P}$-wave onset on electrocardiography just before atrial contraction) and the LAVmin (defined as the volume immediately after mitral closure) were calculated from the apical fourchamber and two-chamber views using the modified Simpson biplane method, followed by the derivation of LA expansion index (LAEI), LA passive emptying fraction (LAPEF), LA active emptying fraction (LAAEF), total LA emptying fraction (TLAEF), as follows: LAEI $=(\mathrm{LAV} \max -\mathrm{LAVmin}) / \mathrm{LAVmin}$ $\times 100 \%$ LAPEF $=(\mathrm{LAV} \max -\mathrm{LAVp}) / \mathrm{LAV} \max \times 100 \%$; $\mathrm{LAAEF}=(\mathrm{LAVp}-\mathrm{LAVmin}) / \mathrm{LAV} \times 100 \%, \mathrm{TLAEF}=(\mathrm{LAV}-$ max-LAV min)/LAV max $\times 100 \%$, which represent LA reservoir function, LA conduit function, LA contractile function and LA overall function, respectively [Rosca 2011]. The pulmonary artery systolic pressure at rest was estimated through assessment of the tricuspid regurgitant jet or right atrial pressure [Lancellotti 2010]. Analysis of echocardiographic parameters was performed retrospectively by another doctor blinded to the research groupings of the patients.

Statistical analysis: All values were expressed as the mean \pm standard error of the mean. Comparisons of the quantitative data between the two groups were tested by independent samples t-test, between multiple groups were tested by 
Table 1. Baseline characteristics of controls and MR population

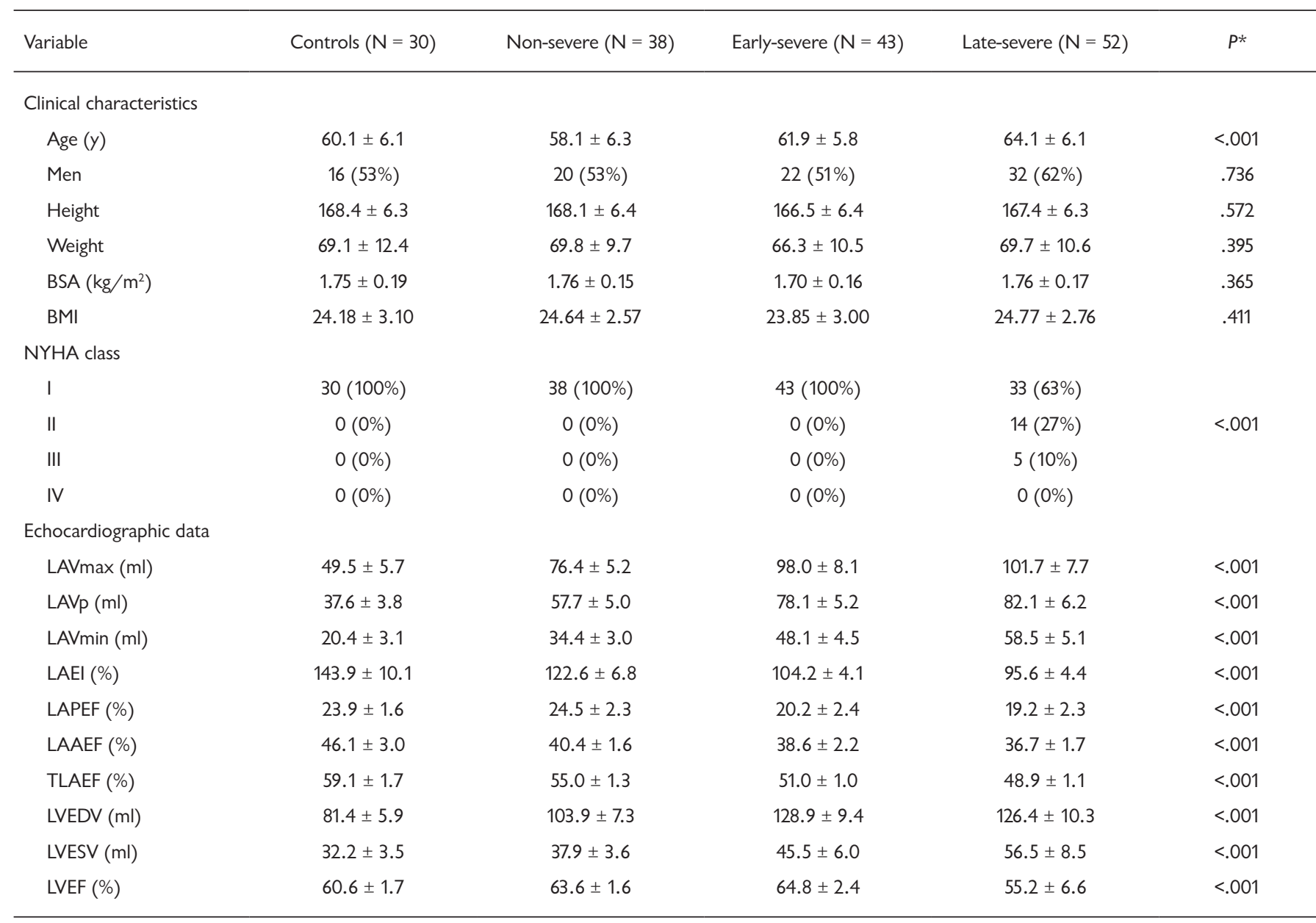

BSA, body surface area; BMI, body mass index; NYHA, New York Heart Association; LAVmax: the left atrial volume immediately prior to mitral valve opening; LAVp: the left atrial volume at P-wave onset on electrocardiography just before atrial contraction; LAVmin: the volume immediately after mitral closure; LAEl: left atrial expansion index; LAPEF: left atrial passive emptying fraction; LAAEF: left atrial active emptying fraction; TLAEF: total left atrial emptying fraction; LVEDV: left ventricular end-diastolic volume; LVESV: left ventricular end-systolic volume; LVEF: left ventricular ejection fraction. *For comparison among control group, non-severe MR group, early-severe MR group, and late-severe MR group

One-Way ANOVA analysis and LSD test. Logistic regression analysis was employed to determine whether LA parameters could predict the indications of mitral valve surgery. Receiver operating characteristic curve analyses were generated for independent indexes to obtain optimal cut-offs. $P<.05$ was considered statistically significant. The software SPSS 24.0 was used in the statistical analysis.

Data availability statement: The datasets generated during and/or analyzed during the current study are available from the corresponding author on reasonable request.

\section{RESULTS}

Patient population: A total of 133 patients who met the inclusion criteria underwent echocardiography in our hospital over a period of two and a half years. The same frame rate for echocardiographic examinations performed was used for echocardiographic examination during the period of two and a half years. Echocardiographic and clinical characteristics of the 133 patients were summarized in Table 1. Overall, preserved LV systolic function was observed in the MR group, with mild LVESV dilatation and LVEDV dilatation. LAV dilated severely, and LAV in the MR group was about twice as large as that in the control group. In 95 patients with severe MR, 43 (45\%) patients did not meet any conventional criteria for mitral surgical operation, while $52(55 \%)$ patients met one or more criteria. Of these patients with surgical indications, $\mathrm{LV}$ end-systolic diameter $>40 \mathrm{~mm}$ in 12 patients (23\%), LVEF $<60 \%$ in $34(65 \%)$, symptoms were present in $19(37 \%)$, and systolic pulmonary arterial pressure at rest $>50 \mathrm{~mm} \mathrm{Hg}$ in 18 (35\%), and no patients presented with LVEFs $<30 \%$.

LAV enlargement: With the increase of MR, the LAV increased gradually (Figure 1C). Compared with the MR 
Table 2. Univariate, multivariate and receiver operating characteristic analysis of left atrial parameters

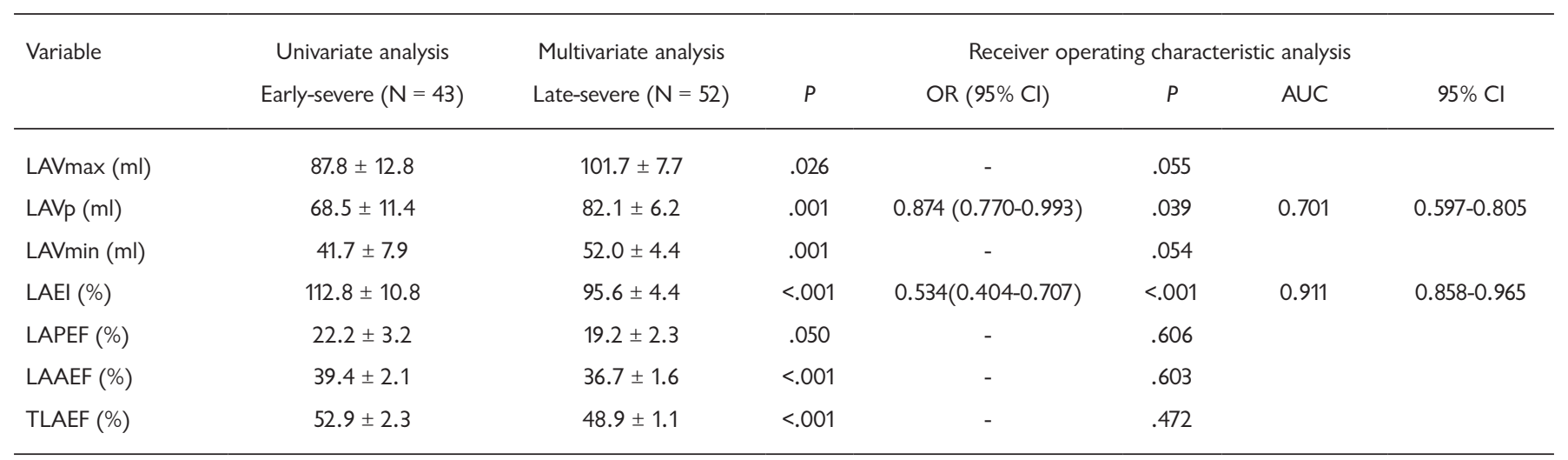

LAVmax: the left atrial volume immediately prior to mitral valve opening; LAVp: the left atrial volume at P-wave onset on electrocardiography just before atrial contraction; LAVmin: the volume immediately after mitral closure; LAEl: left atrial expansion index; LAPEF: left atrial passive emptying fraction; LAAEF: left atrial active emptying fraction; TLAEF: total left atrial emptying fraction; OR: mitral regurgitation; Cl: confidence interval

patients without surgical indication, the LAV was increased in the MR patients with surgical indication. LAVmax, LAVp, and LAVmin showed statistically significant differences between the two groups $(87.7 \pm 12.8$ vs. $101.7 \pm 7.7 \%, P<.001$; $68.5 \pm 11.4$ versus $82.1 \pm 6.2, P<.001 ; 41.7 \pm 7.9$ versus $52.0 \pm$ $4.4, P<.001$, respectively) (Figure $1 \mathrm{~A}$ ).

Left atrial function decrease: With the increase of MR, the left atrial function gradually decreased (Figure 1D). Compared with the MR patients without surgical indication, the parameters reflecting left atrial function were decreased in the MR patients with surgical indication. LAEI, LAPEF, LAAEF, and TLAEF showed statistically significant differences between the two groups $(112.8 \pm 10.8$ versus $95.6 \pm$ $4.4 \%, P<.001 ; 22.2 \pm 3.2$ versus $19.2 \pm 2.3, P<.001 ; 39.4 \pm$ 2.1 versus $36.7 \pm 1.6, P<.001 ; 52.9 \pm 2.3$ versus $48.9 \pm 1.1$, $P<.001$, respectively) (Figure $1 \mathrm{~B}$ ).

Prediction of LA function for surgical indications: Univariate analysis showed that left atrial volume (including LAVmax, LAVp, and LAVmin) and function (including LAEI, LAPEF, LAAEF, and TLAEF) had significant differences between MR patients with mitral valve operation and those without. Multivariate analysis showed that LAVP and LAEI were independent risk factors for mitral operation in patients with MR. The receiver operating characteristic curve analysis was performed to determine the specificity and sensitivity of LAVP and LAEI for predicting mitral valve surgery indications. The results showed that TAEI had the highest predictive value, evidenced by an area under the curve of 0.91 (95\% confidence interval [CI], 0.858-0.965) (Figure 2 and Table 2). In particular, a value of LAEI $\leq 97 \%$ predicted the presence of mitral surgery indications with sensitivity and specificity of $98 \%$ and $67 \%$, respectively.

\section{DISCUSSION}

In this study, we evaluated the volume and function of LA in different degrees of MR. During MR, with the increase of left atrial volume load, the volume of LA gradually increased, while the function of LA gradually decreased. These left atrial volume and function parameters were statistically different not only between the control group and the experimental group, but also between the MR patients with and without surgical indication, which suggests that the LA parameters may be used as indicators of the severity and surgical indications for MR. Our further study showed that LAEI and LAVp were independent risk factors for surgical indications in MR patients, and ultimately LAEI had the highest predictive value.

With the increase in MR, a gradual increase in LAV could be observed in this study, including LAVmax, LAVp, and $\mathrm{LAVmin}$. The reason is that $\mathrm{LV}$ can pump excess blood into the low-pressure LA and increased left atrial pressure and volume load dramatically, which makes the LA the most damaged organ in the early stage. LA dilation in response to MR has been noted for a long period and is thought to be able to predict adverse outcomes in MR. One study is consistent with our results, showing that LA enlargement is a strong and independent predictor of outcome under medical management, and patients with LA index $\geq 60 \mathrm{ml} / \mathrm{m}^{2}$ incur excess mortality and frequent cardiac events whereas those with LA index 30 to $59 \mathrm{ml} / \mathrm{m}^{2}$ tend to present with notable cardiac events [Le Tourneau 2010].

During LV systole, mitral valve closure makes the LA a reservoir to receive pulmonary venous blood flow, which is stored as pressure potential energy. The LA reservoir function is the passive expansion capacity when receiving blood return, which is largely affected by the stiffness of the LA. In an open-pericardium, a paced (70 and $90 \mathrm{bpm}$ ) pig model of LV regional ischemia (left anterior descending coronary constriction), with high-fidelity $\mathrm{LV}$, LA, and right ventricle (RV) pressure recordings, the point has been confirmed [Barbier 1999]. This study showed a negative correlation between degree of MR and LAEI, the index of the LA reservoir function, which may be due to increased left atrial stiffness. Pressure and blood flow shearing force in MR patients can cause chronic inflammatory, interstitial fibrosis and 


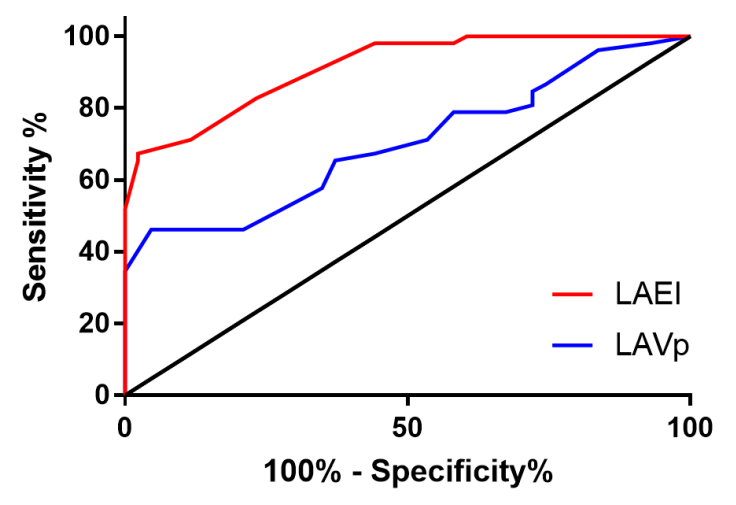

Figure 2. Receiver-operating characteristic curve for prediction of mitral regurgitation with a surgical indication. LAEI: left atrial expansion index; LAVp: the left atrial volume at P-wave onset on electrocardiography just before atrial contraction

myocyte hypertrophy, which are the causes of left atrial stiffness [Moustafa 2011; Corradi 2012]. Studies have found a significant negative correlation between LA reservoir strain and myocardial fibrosis [Cameli 2013], a typical pathological change in MR [Li 2018; Cameli 2013]. These studies share our view that a large variety of ultrastructural changes and degeneration, including interstitial fibrosis and cellular degenerative changes, were present in patients undergoing cardiac surgery for mitral valvular disease. The increase in interstitial fibrosis in the setting of mitral valvular disease compromises the elastic properties of the atrial myocardium and inevitably leads to impairment of atrial compliance and thus to a reduction of LA reservoir function.

LAPEF, reflecting LA conduit function, decreased with the exacerbation of MR in this study. In the early diastolic phase of the $\mathrm{LV}$, the period from mitral valve opening to atrial active contraction (i.e. P-wave front), blood returning from pulmonary venous can flow directly into the $L V$ via the LA, which is the function of the LA as a conduit. Left atrial conduit function is mainly determined by left atrial resilience and LA afterload [Rosca 2011; Pagel 2003]. During MR, increased stiffness of the left atrial myocardium resulting from damage to the myocardial ultrastructure may reduce LA elasticity and affect LA conduit function [Le Tourneau 2010; Moustafa 2011]. In addition, increased early diastolic LV filling pressures may be another important cause of decreased LA conduit function [Zile 1991; Borg 2009]. Although the association between preload, afterload, LA contractile function, and the LA contractile contribution to LV filling is particularly complex, the presence of high LV filling pressures in MR will result in a greater atrial afterload, and thus a decreased atrial contractile contribution.

LAAEF, which is an autonomic contraction of the LA, further inflates the LV during late left ventricular diastole. It is affected by left atrial preload and left atrial systolic capacity, based on the Frank-Starling mechanism [Rosca 2011; Pagel 2003]. Depending on the Frank-Starling mechanism, a properly increased preload can increase contraction. However, this study found that patients with MR, regardless of the degree of regurgitation, showed decreased LAAEF. This may be the overlarge dilation of the LA that cause the sarcomere to exceed the optimum initial length. This study found that the left atrial volume of patients with mild and moderate MR was more than $50 \%$ higher than that of normal patients.

For patients with MR, surgery is an effective treatment. Optimal timing of surgical treatment of MR is crucial and inappropriate timing can reduce the patient's quality of life or long-term survival. Left atrial parameter, which integrates multiple determinants in one variable, provides in one measurement major prognostic information and is essential in the risk-stratification of patients with MR [Messika-Zeitoun 2007]. This makes it possible for the LA index to be a predictor of the timing of the operation. The current guidelines for surgery are based on the severity of MR and its impact on cardiac performance and state of symptoms, including $\mathrm{LV}$ systolic dysfunction, the presence of symptoms, atrial fibrillation, or pulmonary hypertension. These conditions may be secondary to LA dysfunction and remodeling. Enlargement and fibrosis of the LA are the basis of atrial fibrillation; the decrease of LA expansion index directly affects pulmonary venous reflux and becomes the basis of pulmonary hypertension. The decrease of left atrial systolic function can reduce the left ventricular filling and LVEF. Accordingly, LA characteristics may indicate the hemodynamic implications of MR more than conventional criteria for surgical indication at an earlier stage [Messika-Zeitoun 2007]. In organic MR, higher LA index is the combined result of multiple physiological effects, provides independent prognostic information, and therefore should be part of a comprehensive echocardiographic examination.

The further study of left atrial function may give us a better understanding on MR and provide a reference for the timing of the operation. Finally, multivariate analysis was used to screen out independent risk factors from a wide range of parameters in the LA in this study, according to current guidelines. It was found that LAEI and LAVp were independent risk factors for MR surgery, which is consistent with previous studies [Ring 2014; Debonnaire 2013]. Receiveroperating characteristic curve shows that LAEI was more predictable than LAV for MR surgery. However, previous studies suggest that LA reservoir strain has the best predictability [Debonnaire 2013]. We go through different approaches but equal results. Left atrial functions, instead of left atrial volume were shown to be associated with the presence of symptoms and were directly related to atrial stiffness. In addition, left atrial functions were demonstrated to be associated with a higher incidence of atrial fibrillation. The difference may be due to differences in the population between the two studies.

Limitation: The limitations of this study are its consideration of patients only at a single center, and a relatively small sample size. Other limitations are the failure to include all types of MR, such as patients with atrial fibrillation and LVEF less than $30 \%$, and the failure to not provide surgical prognosis but only reporting the relationship between left atrial function and conventional surgical indications. In addition, 
inter-observer variation in measuring echocardiographic parameters was a limitation of the study.

\section{CONCLUSION}

The LA is severely affected during MR. Atrial adaptation is characterized by increasing LAV and decreased LA function. The degree of impairment of atrial function corresponds with the indication for surgical intervention, among which, the LAEI had the highest value to predict the optimum timing of mitral valve operation.

\section{REFERENCES}

Avierinos JF, Gersh BJ, Melton LJ 3rd, Bailey KR, Shub C, Nishimura RA, et al. 2002. Natural history of asymptomatic mitral valve prolapse in the community. Circulation. 106(11):1355-61.

Barbier P, Solomon SB, Schiller NB, Glantz SA. 1999. Left atrial relaxation and left ventricular systolic function determine left atrial reservoir function. Circulation. 100(4):427-36.

Borg AN, Pearce KA, Williams SG, Ray SG. 2009. Left atrial function and deformation in chronic primary mitral regurgitation. Eur J Echocardiogr. 10(7):833-40.

Cameli M, Lisi M, Righini FM, Massoni A, Natali BM, Focardi M, et al. 2013. Usefulness of atrial deformation analysis to predict left atrial fibrosis and endocardial thickness in patients undergoing mitral valve operations for severe mitral regurgitation secondary to mitral valve prolapse. Am J Cardiol. 111(4):595-601.

Corradi D, Callegari S, Maestri R, Ferrara D, Mangieri D, Alinovi R, et al. 2012. Differential structural remodeling of the left-atrial posterior wall in patients affected by mitral regurgitation with or without persistent atrial fibrillation: a morphological and molecular study. J Cardiovasc Electrophysiol. 23(3):271-9.

Debonnaire P, Leong DP, Witkowski TG, Al Amri I, Joyce E, Katsanos S, et al. 2013. Left atrial function by two-dimensional speckle-tracking echocardiography in patients with severe organic mitral regurgitation: association with guidelines-based surgical indication and postoperative (long-term) survival. J Am Soc Echocardiogr. 26(9):1053-62.

Delahaye JP, Gare JP, Viguier E, Delahaye F, De Gevigney G, Milon H. 1991. Natural history of severe mitral regurgitation. Eur Heart J. 12 Suppl B:5-9.

Enriquez-Sarano M, Avierinos JF, Messika-Zeitoun D, Detaint D, Capps M, Nkomo V, et al. 2005. Quantitative determinants of the outcome of asymptomatic mitral regurgitation. N Engl J Med. 352(9):875-83.

Kang DH, Park SJ, Sun BJ, Cho EJ, Kim DH, Yun SC, et al. 2014. Early surgery versus conventional treatment for asymptomatic severe mitral regurgitation: a propensity analysis. J Am Coll Cardiol. 63(22):2398-407.

Lancellotti P, Moura L, Pierard LA, Agricola E, Popescu BA, Tribouilloy C, et al. 2010. European Association of Echocardiography recommendations for the assessment of valvular regurgitation. Part 2: mitral and tricuspid regurgitation (native valve disease). Eur J Echocardiogr. 11(4):307-32.

Le Tourneau T, Messika-Zeitoun D, Russo A, Detaint D, Topilsky Y, Mahoney DW, et al. 2010. Impact of left atrial volume on clinical outcome in organic mitral regurgitation. J Am Coll Cardiol. 56(7):570-8.

Li B, Cui Y, Zhang D, Luo X, Luo F, Li B, et al. 2018. The characteristics of a porcine mitral regurgitation model. Exp Anim. 67(4):463-77.

Messika-Zeitoun D, Bellamy M, Avierinos JF, Breen J, Eusemann C, Rossi A, et al. 2007. Left atrial remodelling in mitral regurgitation--methodologic approach, physiological determinants, and outcome implications: a prospective quantitative Doppler-echocardiographic and electron beamcomputed tomographic study. Eur Heart J. 28(14):1773-81.

Moustafa SE, Alharthi M, Kansal M, Deng Y, Chandrasekaran K, Mookadam F. 2011. Global left atrial dysfunction and regional heterogeneity in primary chronic mitral insufficiency. Eur J Echocardiogr. 12(5):384-93.

Nishimura RA, Otto CM, Bonow RO, Carabello BA, Erwin JP 3rd, Fleisher LA, et al. 2017. 2017 AHA/ACC Focused Update of the 2014 AHA/ACC Guideline for the Management of Patients With Valvular Heart Disease: A Report of the American College of Cardiology/American Heart Association Task Force on Clinical Practice Guidelines. J Am Coll Cardiol. 70(2):252-89.

Nkomo VT, Gardin JM, Skelton TN, Gottdiener JS, Scott CG, Enriquez-Sarano M. 2006. Burden of valvular heart diseases: a population-based study. Lancet. 368(9540):1005-11.

Pagel PS, Kehl F, Gare M, Hettrick DA, Kersten JR, Warltier DC. 2003. Mechanical function of the left atrium: new insights based on analysis of pressure-volume relations and Doppler echocardiography. Anesthesiology. 98(4):975-94.

Ring L, Rana BS, Wells FC, Kydd AC, Dutka DP. 2014. Atrial function as a guide to timing of intervention in mitral valve prolapse with mitral regurgitation. JACC Cardiovasc Imaging. 7(3):225-32.

Rosca M, Lancellotti P, Popescu BA, Piérard LA. 2011. Left atrial function: pathophysiology, echocardiographic assessment, and clinical applications. Heart. 97(23):1982-9.

Zile MR, Tomita M, Nakano K, Mirsky I, Usher B, Lindroth J, et al. 1991. Effects of left ventricular volume overload produced by mitral regurgitation on diastolic function. Am J Physiol. 261(5 Pt 2):H1471-80. 